\section{Preventing Blood Contamination in Nuclear Pharmacies: Lessons from an Outbreak of Hepatitis C Virus Infections and Contaminated 99mTc-Sestamibi}

TO THE EDITOR: We thank Dr. Hung for his interest in our recent article and for highlighting its relevance to the nuclear medicine community $(1,2)$. We agree that understanding failures that can lead to pharmaceutical contamination and subsequent disease transmission is paramount to prevention. Hung states that knowledge of the exact breach that led to hepatitis $\mathrm{C}$ virus (HCV) transmission in the setting of radiopharmaceutical preparation is necessary to prevent recurrences. In the nuclear pharmacy we investigated, multiple deficiencies in practices were identified, any of which might have contributed to the HCV transmission event. Each of these practices represents an opportunity for contamination and should be corrected. In the following response, we hope to clarify the pharmacy practices described in the article and support our assertion that a broad prevention strategy—beginning with a critical examination of current practices and gaps in oversight and training-is needed to reduce the risk of blood contamination in nuclear pharmacies.

During the white blood cell (WBC) radiolabeling procedure involving blood from the HCV-infected source patient, the pharmacist had to exit the blood room and use the calibration hood in the main room because there was no dose calibrator in the blood room. The ${ }^{111}$ In was calibrated in the main room and brought back to the blood room to be mixed with WBCs and plasma. Later in the process, the final syringe containing radiolabeled WBCs was also calibrated in the main room. In addition, we learned that pharmacists assigned to the blood room frequently would answer phone calls in the main room to receive prescription orders. Each of these movements between rooms was an opportunity for contaminated equipment to inadvertently be carried out of the blood room and left in the main room, where ${ }^{99 \mathrm{~m}} \mathrm{Tc}$-sestamibi was prepared.

Only doses from the first ${ }^{99 \mathrm{~m}} \mathrm{Tc}$-sestamibi vial prepared on October 15, 2004 (vial 1), resulted in HCV infections. All patients who received doses from any of the 5 vials prepared immediately after vial 1 were advised to undergo testing. Although not all agreed to be tested, $81 \%$ were tested, and results were negative, strongly supporting our conclusion that only vial 1 was contaminated. There are several possible explanations. A contaminated vial or bag of saline could have been used to dilute all the ${ }^{99 \mathrm{~m}} \mathrm{Tc}-$ sestamibi vials (vials 1-6) before the heating step (which would have killed the virus). For postheating dilution, it is possible that enough saline remained in the contaminated vial or bag to dilute vial 1 but not vials 2-6 (the vials were prepared in numeric order). For postheating dilution, the contents of each ${ }^{99 \mathrm{~m}} \mathrm{Tc}$-sestamibi vial were transferred to a larger vial to accommodate the added volume of saline. Each transfer involved a separate syringe; the syringes were not shared between vials because of their radioactive contents. Thus, it is possible that the syringe used to transfer the contents of vial 1 was contaminated and did not affect the other vials. There were other syringes and steps in the process that

COPYRIGHT @ 2007 by the Society of Nuclear Medicine, Inc. might have affected only one vial-for example, the syringe used to draw a quality control sample-however, it seems unlikely that this would have resulted in contamination.

As mentioned in the article, laminar flow workstations throughout the pharmacy were stocked with unwrapped syringes for convenience and ease of use. It would be difficult to know if a syringe lying in a workstation or on a counter was used or unused; a small amount of serum in the syringe or hub could easily go unnoticed. No pharmaceuticals were prepared in the main-room hood (where vials 1-6 were prepared) between 1 PM on October 14 and 1:05 AM on October 15. No pharmacists reported improper disposal or reuse of contaminated equipment or supplies. Individual patient doses were drawn by pharmacy technicians in different workstations, not the one where vials 1-6 were prepared; however, these workstations were in the same main room.

The proposed revisions to U.S. Pharmacopeia (USP) $<797>$ highlighted by Hung stemmed from our investigation of this event and the resultant communications. We support the revisions regarding separation of blood from compounded sterile preparation (CSP) areas and recommend more detailed guidance to clarify the standards. For example, use of a dose calibrator within a CSP area for blood preparations does not constitute complete physical separation, nor does a blood preparation area that is separated from the CSP area by an open entryway. During a given shift, staff should be assigned to activities housed entirely within the blood preparation area or within the CSP area, with no duties that would result in traffic between areas. Each area should have dedicated supplies, equipment, and waste disposal to eliminate sharing of these items or overlap in pathways. Blood preparations should also be completely separated from one another to prevent crosscontamination. Blood products from more than one patient should never be manipulated at the same workstation.

Hung notes that both the Nuclear Pharmacy Compounding Guidelines and the American Pharmacists Association (APhA) support adherence to the USP General Chapter $<1206>$ guidance for compounding high-risk radiopharmaceuticals, including radiolabeled WBCs. The risk classification system focuses on the risk of microbial contamination or other adverse outcomes resulting from improper preparation or storage of a pharmaceutical; higher-risk preparations require heightened precautions. Although radiolabeled WBCs are considered high-risk in this classification, other pharmaceuticals prepared in the vicinity of blood products are not. The risk level for many radiopharmaceuticals appears to be subjective (3). In a letter to the USP, the APhA suggested that certain "short-lived" radiopharmaceuticals should be considered low-risk because of their same-day administration, regardless of how they are compounded (4). The proposed revisions to USP $<797>$ state, "the following shall be designated Low-Risk Level CSPs: (1) radiopharmaceutical dosage units with volumes of $15 \mathrm{~mL}$ and less and expiration times of 18 hours and shorter, such as those prepared from eluates from technetium-99m/molybdenum 99 generator systems" (5). Unfortunately, these short-lived products do not have a lower risk of viral transmission. APhA also commented that "environmental controls are insignificant compared to operator technique, where operator technique is grounded in training and supported by sterility testing (e.g., media fills)" (4). We agree with the importance of operator technique, but media-fill challenge tests and similar quality 
assurance measures do not detect viral contamination, and there are currently no requirements for operator training on blood-borne pathogen contamination. Professional and regulatory organizations should provide explicit guidance on appropriate precautions for commonly prepared radiopharmaceuticals and should consider whether additional guidance is needed for all sterile pharmaceuticals prepared in pharmacies that handle blood.

In summary, the current guidelines cited by Hung do not adequately address the risk of blood contamination of pharmaceuticals in nuclear pharmacies. The introduction of blood products into nuclear pharmacies where sterile pharmaceuticals are prepared should be accompanied by aggressive efforts to ensure safe blood-handling practices and appropriate infection control. Pharmacists and technicians working in these settings should have a thorough understanding of precautions to prevent blood contamination and how these differ from the approach to bacterial or fungal contamination and growth. Increased awareness of the risks of blood-borne pathogens in nuclear pharmacies through enhanced training, education, and professional leadership is needed. Nuclear pharmacies that handle blood or any potentially infectious biologic material should adhere to higher standards to ensure product integrity and patient safety.

DISCLAIMER: The findings and conclusions in this letter are those of the authors and do not necessarily represent the views of the Centers for Disease Control and Prevention.

\section{REFERENCES}

1. Hung JC. Hepatitis $C$ virus infections from contaminated ${ }^{99 \mathrm{~m}} \mathrm{Tc}$-sestamibi [letter]. J Nucl Med. 2007;48:847-848.

2. Patel PR, Larson AK, Castel AD, et al. Hepatitis C virus infections from a contaminated radiopharmaceutical used in myocardial perfusion studies. JAMA. 2006;296:2005-2011.

3. Hung JC. The potential impact of USP general chapter $<797>$ on procedures and requirements for the preparation of sterile radiopharmaceuticals. $\mathrm{J} \mathrm{Nucl} \mathrm{Med}$. 2004;45(6):21N-26N.

4. American Pharmacists Association web site. APhA comments on USP chapter 797. Available at: http://www.aphanet.org/AM/Template.cfm?Section=Home\& CONTENTID $=6704 \&$ TEMPLATE $=/ \mathrm{CM} /$ ContentDisplay.cfm. Accessed August $21,2007$.

5. U.S. Pharmacopeia web site. $<797>$ Pharmaceutical Compounding: Sterile Preparations. Available at: http://www.usp.org/pdf/EN/USPNF/PF797.pdf. Accessed August 21, 2007

Priti R. Patel Joseph F. Perz Anthony E. Fiore Centers for Disease Control and Prevention Atlanta, Georgia

Amanda D. Castel

George Washington University School of Public Health and Health Services Washington, DC

DOI: 10.2967/jnumed.107.045195

REPLY: It seems to me that the letter by Patel et al. failed to identify the specific cause for this hepatitis $\mathrm{C}$ virus (HCV) outbreak, and in fact the letter made the whole issue more confusing. If the pharmacist who radiolabeled WBCs on October 14, 2004, had to exit the "blood room" to the "main room" to measure the radioactivity of ${ }^{111} \mathrm{In}$ and ${ }^{111} \mathrm{In}$-oxine-labeled white blood cells, the syringes containing these materials would have been capped during the dose measurement and brought back to the blood room after the measurement. Also, the letter indicated that these syringes would not be reused "because of their radioactive contents." Thus, the process and syringes should not be the causes for the HCV contamination. Patel et al. surmised that a contaminated vial or bag of saline or possibly a contaminated syringe was somehow moved from the blood room to the main room and somehow caused contamination only to vial 1 and not the other 5 vials of ${ }^{99 \mathrm{~m}} \mathrm{Tc}-$ sestamibi, all of which were prepared $1 \mathrm{~min}$ apart by the same pharmacist in the same hood (1). Let us assume that contaminated saline vials/bags or syringes were carried inadvertently into the main room on the afternoon of October 14, 2004. Before the preparation of ${ }^{99 \mathrm{~m}} \mathrm{Tc}$-sestamibi (vials 1-6) in the early morning of October 15, 2004, the pharmacist would have discarded any contaminated supplies (e.g., unwrapped syringes or used saline vials or bags) left in the hood of the main room as per the statement (i.e., "No pharmacists reported improper disposal or reuse of contaminated equipment or supplies.") in the letter by Patel et al.

Even though $59(82 \%)$ of 72 patients who were injected with 99m Tc-sestamibi drawn from vials 2-6 were later tested for anti$\mathrm{HCV}$ and all were negative (1), I think that it would be prudent to closely follow up the medical condition of the other 13 individuals who did not take part in this test because the exact cause for this catastrophic HCV contamination is still unclear. Have symptoms related to HCV, hepatitis B virus, or HIV (human immunodeficiency virus) developed in any of these 13 patients after the incident?

There is no doubt that higher standards should be established for the handling of radiolabeled blood cells to ensure product integrity and patient safety. However, we should carefully evaluate the suitability and practicality of any proposed standards so that their cost (e.g., the cost of significant changes in remodeling, monitoring, or staffing) does not force facilities (especially small or rural nuclear pharmacies/nuclear medicine laboratories) to discontinue providing products such as ${ }^{111}$ In-oxine-labeled white blood cells to patients.

\section{REFERENCE}

1. Patel PR, Larson AK, Castel AD, et al. Hepatitis $C$ virus infections from a contaminated radiopharmaceutical used in myocardial perfusion studies. JAMA. 2006;296:2005-2011.

\section{Attenuation Correction for Stress and Rest PET ${ }^{82} \mathrm{Rb}$ Myocardial Perfusion Images}

TO THE EDITOR: The July 2007 article by Gould et al. (1) reported a $40 \%$ false-positive rate for cardiac PET ${ }^{82} \mathrm{Rb}$ myocardial perfusion imaging with $\mathrm{CT}$ attenuation correction, using helical slow imaging ( $29 \mathrm{~s}$ ) during free breathing and helical fast imaging (4s) during a breath-hold at end expiration. Further, the authors suggested that correction with nonhelical, time-averaged 